\title{
Analgesic Regimen and Readmission Following Tonsillectomy
}

\author{
Lyudmila Kishikova ${ }^{1}$, Matthew D. Smith ${ }^{1}$, Jason C. Fleming ${ }^{2}$, Michael O’Connell ${ }^{2}$ \\ ${ }^{1}$ Brighton and Sussex Medical School, Brighton, UK \\ ${ }^{2}$ ENT Department, Brighton and Sussex University Hospitals Trust, Brighton, UK \\ Email: l.kishikova@uni.bsms.ac.uk
}

Received April 22, 2013; revised May 23, 2013; accepted June 15, 2013

Copyright (C 2013 Lyudmila Kishikova et al. This is an open access article distributed under the Creative Commons Attribution License, which permits unrestricted use, distribution, and reproduction in any medium, provided the original work is properly cited.

\begin{abstract}
Objective: To define the analgesic regimen given following tonsillectomy in a large ENT department and correlate this with readmission for secondary complications. Methods: We performed a retrospective case note review of patients undergoing tonsillectomy within a six month period. Demographic information and relevant case information was collected including operative details, discharge medication and readmission details. Results: 125 patients underwent tonsillectomy during the period. 17 different post-operative analgesic regimens were identified with the most common being a paracetamol and ibuprofen combination (26.4\%). 13 patients (10.4\%) were readmitted following discharge from hospital post-operatively, four (3.2\%) for issues related to pain. There was no correlation between analgesic regimens and readmission. Conclusion: No apparent link between readmission and analgesic regimen was identified. The vast variation of analgesic regimens used has prompted development of a formal step-based analgesic protocol.
\end{abstract}

Keywords: Tonsillectomy; Analgesia; Post-Operative Complications

\section{Introduction}

Tonsillectomy is one of the most commonly performed surgical procedures in both adults and children [1]. Although generally a safe and routine procedure, complications of severe pain, haemorrhage and infection can occur. Post-operative pain after tonsillectomy can be of a significant degree and analgesia administered is frequently inadequate. Occasionally, readmission is warranted in cases of severe post-operative pain following discharge, with considerable discomfort and inconvenience for the patient, as well as economic costs for the healthcare institution in general. There is considerable interest in this topic with a recent national UK Clinical Research Network ENT survey having been circulated. It is imperative to understand what factors determine readmission so that this may be predicted and prevented. Within our department, concern about patient readmissions pre-dating the above national survey, as well as the economic implications of such episodes, led to instigation of a survey to assess post-tonsillectomy pain readmissions and specific analgesic prescribing patterns.

\section{Materials and Methods}

Permission was provided by the Trust Clinical Effective- ness department to undertake the survey. This study was a retrospective case note review of patients undergoing tonsillectomy between February and August 2011 within Brighton and Sussex University Hospitals NHS Trust. All patients undergoing tonsillectomy with or without adjunct procedures (e.g. adenoidectomy, grommet insertion) were included. There were no exclusion criteria. Data was obtained from case notes including age, gender, operation type and indication, discharge medication and details of any post-operative readmission. This was entered into a Microsoft ${ }^{\circledR}$ Access 2007 (Redmond, WA) database to allow analysis. Readmission and complication rate was compared to national audit data from the ENT BAO study [1]. Statistics were carried out using a Mann Whitney U test with SPSS v19 (IBM, New York).

\section{Results}

\subsection{Demographics}

125 patients underwent procedures involving tonsillectomy between February and August 2011. Patient ages ranged 3 months -65 years (mean $=13.8$ years). The distribution of ages was $26.4 \%<5$ years, $33.6 \% 5-16$ years and $39.2 \%>16$ years old. This was compared to $15.2 \%, 47.3 \%$ and $37.5 \%$ respectively in the national 
ENT-BAO audit. In our cohort, $37.6 \%$ were male and $62.4 \%$ female.

Of these 125 patients, 79 underwent a standard tonsillectomy (63.2\%), 36 adenotonsillectomy (28.8\%) and 10 adenotonsillectomies with insertion of grommets. Bipolar dissection was used in 111 cases (88.8\%) and cold steel dissection in 14 cases (11.2\%). Figure 1 demonstrates the indications for surgery, of which there were 143 owing to multiple indications in some cases. Recurrent tonsillitis was by far the most common indication, followed by sleep related breathing disorder.

\subsection{Discharge Medication}

Medication prescribed on discharge was analysed, obtained from discharge summary documentation present in case notes. Of the 125 patients, 97 patients were prescribed medication on discharge (77.6\%), with 17 having no medication prescribed on their discharge summaries (13.6\%). For the remaining 11 patients (8.8\%), the discharge summary was either not present in case note sets or was illegible.

The most commonly given analgesic drug was paracetamol, followed by ibuprofen and codeine phosphate (Figure 2(a)). Co-codamol (8/500 and 30/500 formulations), co-dydramol and diclofenac were also given in smaller numbers. A proportion of patients were given benzydamine mouth wash. These drugs were combined in varying combinations, resulting in 17 different discharge analgesic regimens (Figure 2(b)). The most common regimen was concurrent paracetamol and ibuprofen, followed closely by paracetamol, ibuprofen and codeine; other regimens were given in relatively smaller numbers.

\subsection{Readmission}

13 patients were readmitted with secondary complications out of the 125 patients (10.4\%). Of these, four were due to pain (30.8\% of readmissions). This compares to a national average readmission rate of $3.9 \%$, of which $23 \%$ are due to pain [1]. The mean age of readmitting patients was 17.4 years, which increased to 19.3 years when solely considering those readmitted for pain. These findings are summarised in Table 1; 10 out of 13 patients were prescribed discharge medication (76.9\%), with the 3 patients not prescribed medication in the paediatric age range (mean age 7.3 years). Interestingly, the medication regimens of readmitted patients were typically towards the stronger end of the spectrum, with all patients except one readmitting due to pain prescribed an NSAID, paracetamol and codeine preparation as well as benzydamine mouthwash. With these patients all in the above 16 age group, the remaining patient readmitted due to pain was three years old and formally prescribed no discharge medication.

\section{Discussion}

This study concurs with the results of the 2005 ENTBAO national audit, demonstrating that there is a considerable number of readmissions due to pain, with rates of $30.8 \%$ and $23 \%$ of total readmissions respectively. Up for question is whether these are preventable or not, and whether patients at risk of readmitting can be identified before discharge with increased analgesia given for pain prophylaxis. This is particularly important in light of imminent UK Department of Health rules imposing fi-

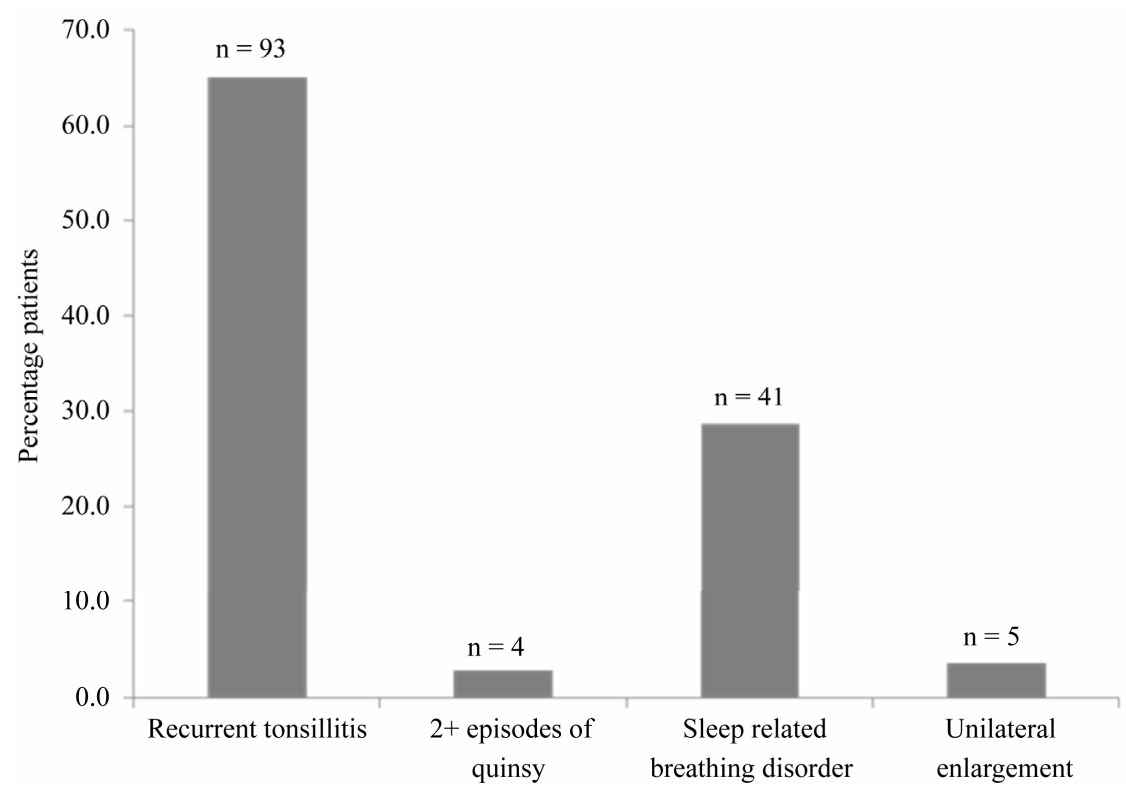

Figure 1. Graph showing indications for surgery. A total of 143 indications were present, in a population of 125 patients, owing to some cases having multiple indications. 


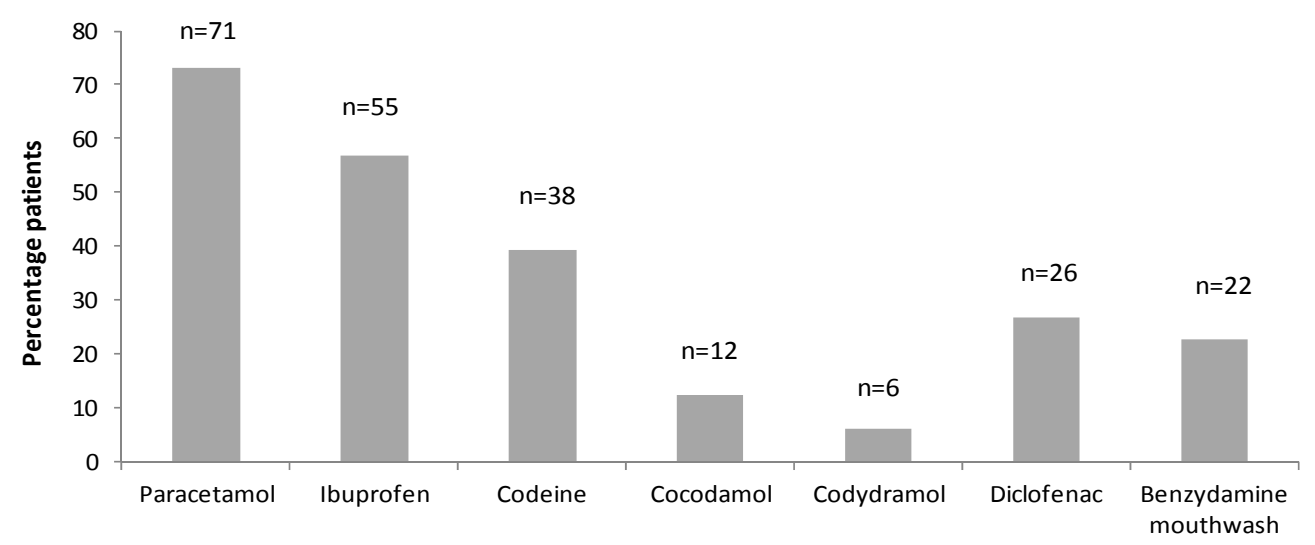

(a)

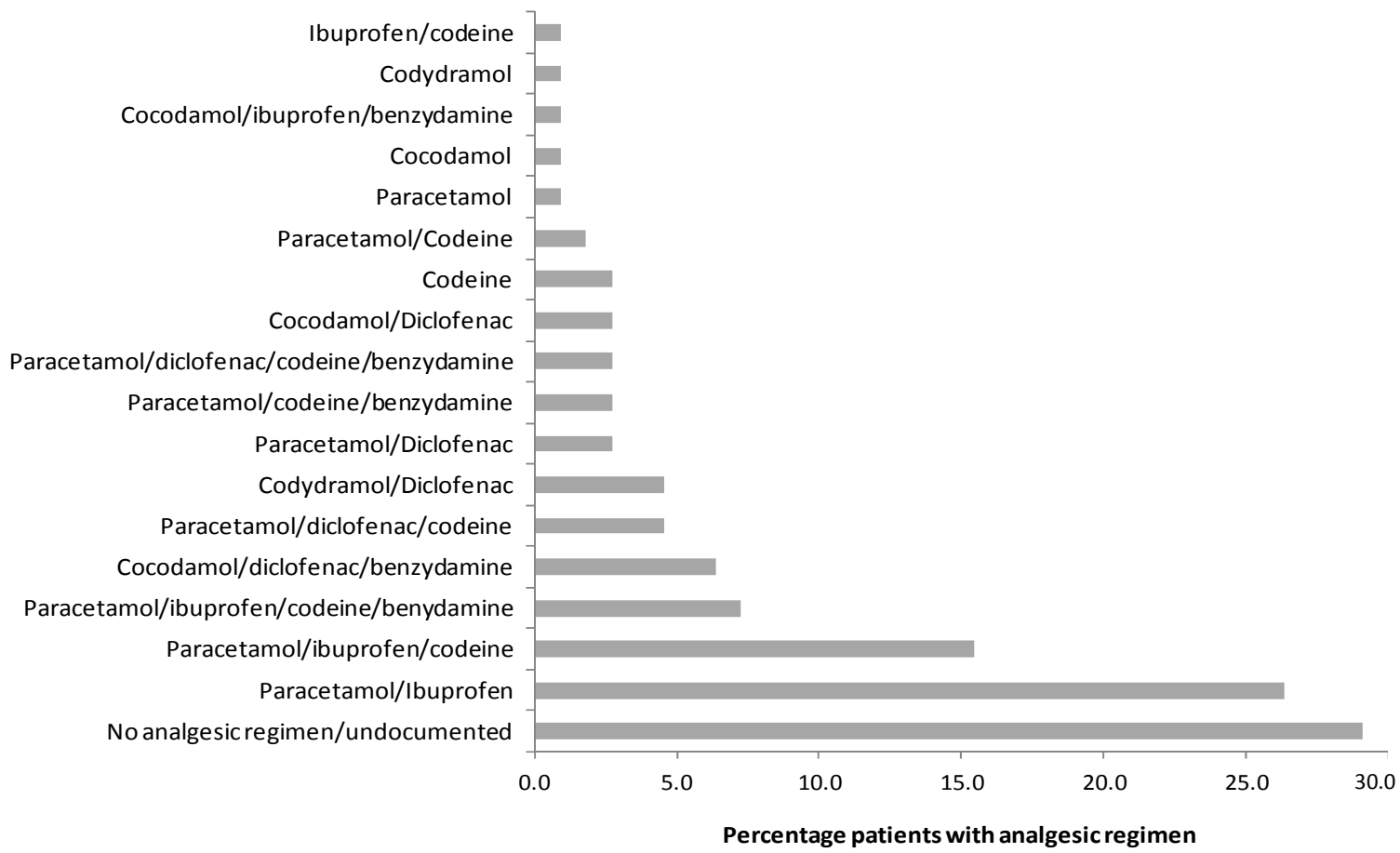

(b)

Figure 2. (a) Graph showing percentage patients receiving each type of analgesic drug. A total of 230 drugs were given to the 125 patients, combined in different regimens; (b) Graph showing the 17 different analgesic drug regimens prescribed on discharge.

Table 1. Details of the four patients readmitting following discharge due to pain. $\mathrm{Rt}=$ recurrent tonsillitis.

\begin{tabular}{cclll}
\hline Age (years) & Indication & Operation & Analgesic regimen & Reason for readmission \\
\hline 18 & Rt & Tonsillectomy (bipolar) & $\begin{array}{l}\text { Paracetamol, diclofenac, } \\
\text { codeine, benzydamine }\end{array}$ & Pain \\
3 & Rt & Tonsillectomy (bipolar) & None & Pain \\
25 & Rt & Tonsillectomy (bipolar) & $\begin{array}{l}\text { Paracetamol, ibuprofen, } \\
\text { codeine, benzydamine } \\
\text { Cocodamol, diclofenac, } \\
\text { benzydamine }\end{array}$ & Pain, dysphagia, neck swelling \\
& Rt & Tonsillectomy (bipolar) & Pain & \\
\hline
\end{tabular}

nancial penalties on Trusts for patient readmissions. Our study attempted to analyse readmissions and ascertain whether there is any pattern associated with these pa- tients, particularly those readmitting due to pain. A majority of those patients readmitted were older than the mean age of the patient population, although no signifi- 
cant difference was found. With the overall mean cohort age in our study higher than the national average, this is one possible explanation for the higher readmission rate experienced in our centre during the study period than the national average.

There was no trend in operation type or indication for those readmitting for any reason (including pain), with these closely reflecting the overall cohort. However, all readmitting patients were discharged on medication regimens that were amongst the stronger regimens given as a cohort, particularly in those readmitting due to pain. At this point, it is not clear whether this is due to the general prescribing habits, or whether these patients were clearly in a greater amount of pain when it came to planning discharge. If the latter were the case, it stands to reason that it would be possible to predict readmission risk by relative post-operative pain levels; that these patients could be prescribed yet stronger analgesia and perhaps monitored in the community post-operatively. It is likely that readmission is due to intrinsic patient factors pertaining to actual pathological/anatomical differences or operative technique, reflected in a greater need for analgesia. We found a trend for readmitting patients to be older, but this did not reach significance. One concept that is not well delineated is how many patients attend their GP in the weeks following tonsillectomy complaining of pain. A recent study indicates that in children, the general practitioner consultation rate following tonsillectomy was over 50\% [2]. Should further studies corroborate this burden on GP appointments, a major healthcare economic saving could be made by ensuring discharge medication is more generous, reducing the need for such consultations.

Patients included in our study received a vast range of analgesic combinations, potentially creating confusion for nursing staff and preventing analgesic use from being a clear proxy of pain levels. Of note, a considerable number of patients were not prescribed analgesia. The majority of these patients were paediatric cases, in which case it was usual practice for the parents to be instructed to use over the counter analgesic preparations, although this advise was not protocol driven, and supplied on an ad hoc individual basis. Benefit would be gained from this being documented for parental education.

Little evidence exists on what medication regimens may be useful, nor what factors should influence choice of drug, both peri- and post-operatively. Homer et al. 2002 suggested that peri-operative analgesic use correlates with improved post-operative pain scores [3]. However, a recent FDA alert has also been issued to warn of the potential risk of specific analgesic use linked to posttonsillectomy use [4]. Life-threatening adverse events and death have occurred in certain children who received codeine after tonsillectomy and/or adenoidectomy for obstructive sleep apnoea syndrome and the alert warns clinicians about the prevalence, ethnic variations and effects of CYP2D6 mutations resulting in "ultra-rapid metabolizers" of substrates of cytochrome P450 2D6, including codeine.

Although this is a relatively small study with a heterogenous patient group, we believe the significant range of analgesic regimens described is reflective of wider prescribing habits. Adverse incidents and readmission rates require urgent discussion within an appropriate forum to highlight this topic and formulate national guidance on appropriate analgesic protocols, whilst maintaining flexibility for clinicians based on local practices, pharmacy preferences and regional ethnic variations.

\section{Conclusion}

Patients were discharged on a vast number of analgesic regimens in our study, and we have identified the need to develop and appraise a logical step-wise approach to post-operative analgesia. Whilst inadequate analgesia did not appear to be a factor for readmission due to pain in this study, national guidelines to help standardise analgesic prescriptions would be helpful for both clinicians and patients/parents alike and help to avoid prescribing related adverse events.

\section{Summary}

- Readmission, including due to pain, is still necessary following tonsillectomy;

- Readmission due to pain does not appear to be caused by inadequate analgesia;

- Patients requiring stronger post-operative analgesic regimens may be at higher risk of readmitting;

- A vast number of different analgesic regimens are given to patients on discharge;

- A stepwise analgesic regimen may be useful in managing post-operative pain on discharge.

\section{REFERENCES}

[1] Royal College of Surgeons of England/British Association of Otorhinolaryngologists, "National Prospective Tonsillectomy Audit,” 2005.

[2] D. W. Stewart, P. G. Ragg, S. Sheppard and G. A. Chalkiadis, "The Severity and Duration of Postoperative Pain and Analgesia Requirements in Children after Tonsillectomy, Orchidopexy, or Inguinal Hernia Repair,” Paediatric Anaesthesia, Vol. 22, No. 2, 2012, pp. 136-143. doi:10.1111/j.1460-9592.2011.03713.x

[3] J. J. Homer, J. D. Frewer, J. Swallow and P. Semple, “An Audit of Post-Operative Analgesia in Children Following Tonsillectomy," The Journal of Laryngology and Otology, Vol. 116, No. 5, 2002, pp. 367-370. doi:10.1258/0022215021910807 
[4] FDA Drug Safety Communication, “Codeine Use in Certain Children after Tonsillectomy and/or Adenoidectomy May Lead to Rare, but Life Threatening Adverse Events or Death,” 2013.

http://www.fda.gov/Drugs/DrugSafety/ucm313631.htm 